\title{
Avifauna de Frederico Westphalen, Rio Grande do Sul, Brasil
}

\author{
Eli Maria Teixeira ${ }^{1}$ \\ Itiberê Piaia Bernardi \\ Fábio André Facco Jacomassa ${ }^{3 *}$ \\ ${ }^{1}$ Programa de Pós-Graduação em Biotecnologia e Gestão Ambiental \\ Faculdades Integradas de Santo Augusto, Santo Augusto - RS, Brasil \\ ${ }^{2}$ Programa de Pós-Graduação em Ecologia \\ Universidade Federal do Paraná, Curitiba - PR, Brasil \\ ${ }^{3}$ Laboratório de Ornitologia e Animais Marinhos, Bloco D (segundo andar), Centro 2 \\ Universidade do Vale do Rio dos Sinos \\ Av. Unisinos, 950 - B, CEP 93.022-000, São Leopoldo - RS, Brasil \\ *Autor para correspondência \\ fabioafj@gmail.com
}

Submetido em 05/03/2009

Aceito para publicação em 12/09/2009

\section{Resumo}

Entre os anos de 2003 e 2008, esforços foram conduzidos para amostrar a avifauna do município de Frederico Westphalen no extremo norte do estado do Rio Grande do Sul. Foram registradas 165 espécies pertencentes a 51 famílias, correspondendo a $26,4 \%$ da riqueza conhecida para o estado.

Unitermos: aves, Floresta Estacional Decidual, fragmentos florestais

\section{Abstract}

Birds of Frederico Westphalen, Rio Grande do Sul, Brazil. From 2003 to 2008, efforts were made to sample the avifauna in Frederico Westphalen, in the extreme north of the state of Rio Grande do Sul. There were 165 species recorded, belonging to 51 families and accounting for $26.4 \%$ of the richness known to the state.

Key words: birds, Deciduous Seasonal Forest, forest fragments

\section{Introdução}

O Rio Grande do Sul é o estado mais austral do Brasil e ocupa uma zona de transição que abrange a ponta sulina das montanhas e matas costeiras do sudeste do país e o limite norte dos campos abertos e ondulados típicos do Uruguai. O estado possui uma diversidade geográfica que ainda contempla praias atlânticas, às manchas de terra de tipo chaquenho no extremo oeste, e às matas do interior ao longo do curso superior do rio Uruguai. A avifauna da região reflete essa variedade (Belton, 1984).

De acordo com Bencke (2001), 624 espécies de aves possuem registros de ocorrência no Rio Grande do Sul. Para a Floresta Estacional Decidual do extremo norte do estado são conhecidas 247 espécies de aves, principalmente a partir dos estudos realizados no 
Parque Estadual do Turvo, município de Derrubadas (Albuquerque, 1981; Mähler Jr., 1996).

O presente trabalho teve por objetivo listar as espécies de aves no município de Frederico Westphalen, extremo norte do estado do Rio Grande do Sul.

\section{Material e Métodos}

\section{Área de estudo}

Frederico Westphalen $\left(27^{\circ} 21^{\prime} \mathrm{S}\right.$ e $\left.53^{\circ} 23^{\prime} \mathrm{W}\right)$ localiza-se na região do Médio Alto Rio Uruguai, a $522 \mathrm{~m}$ de altitude, no estado do Rio Grande do Sul. O clima da região é subtropical do tipo Cfa segundo a classificação de Köppen, onde a temperatura média anual fica em torno $18^{\circ} \mathrm{C}$, com máximas no verão podendo atingir $41^{\circ} \mathrm{C}$ e mínimas no inverno com valores inferiores a $0^{\circ} \mathrm{C}$. A precipitação média anual é elevada, geralmente entre 1800 e $2100 \mathrm{~mm}$, bem distribuída ao longo do ano (Bernardi et al., 2007).

O município apresenta uma paisagem formada por um mosaico de pequenos fragmentos florestais (poucos com mais de 100ha) inseridos em uma matriz caracterizada pela agricultura. Originalmente a mata do
Alto Uruguai se estendia ao longo das bacias dos rios Paraná e Uruguai e ocupava toda essa região, porém em consequência da desenfreada devastação verificada nas últimas décadas pelo tipo de agricultura ali desenvolvida (Irgang, 1980), esta mata está agora fragmentada.

A área de estudo está localizada entre os três grandes remanescentes florestais que restam no extremo norte do estado (Figura 1), distando cerca de $40 \mathrm{~km}$ do Parque Estadual do Turvo (17.491 ha), $28 \mathrm{~km}$ da Terra Indígena de Nonoai (17.000ha) e 14km da Terra Indígena do Guarita (14.740ha) (Bernardi et al., no prelo).

\section{Obtenção dos dados}

Entre janeiro de 2003 e abril de 2008 foram realizados contínuos esforços não sistematizados objetivando registrar a composição da avifauna ocorrente no município.

As aves foram registradas a partir de observação direta com vista desarmada ou com auxílio de binóculos, além de registro de vocalizações. Utilizou-se playback para confirmação da ocorrência de algumas espécies. Aves encontradas mortas com procedência conhecida também foram consideradas nestes registros.

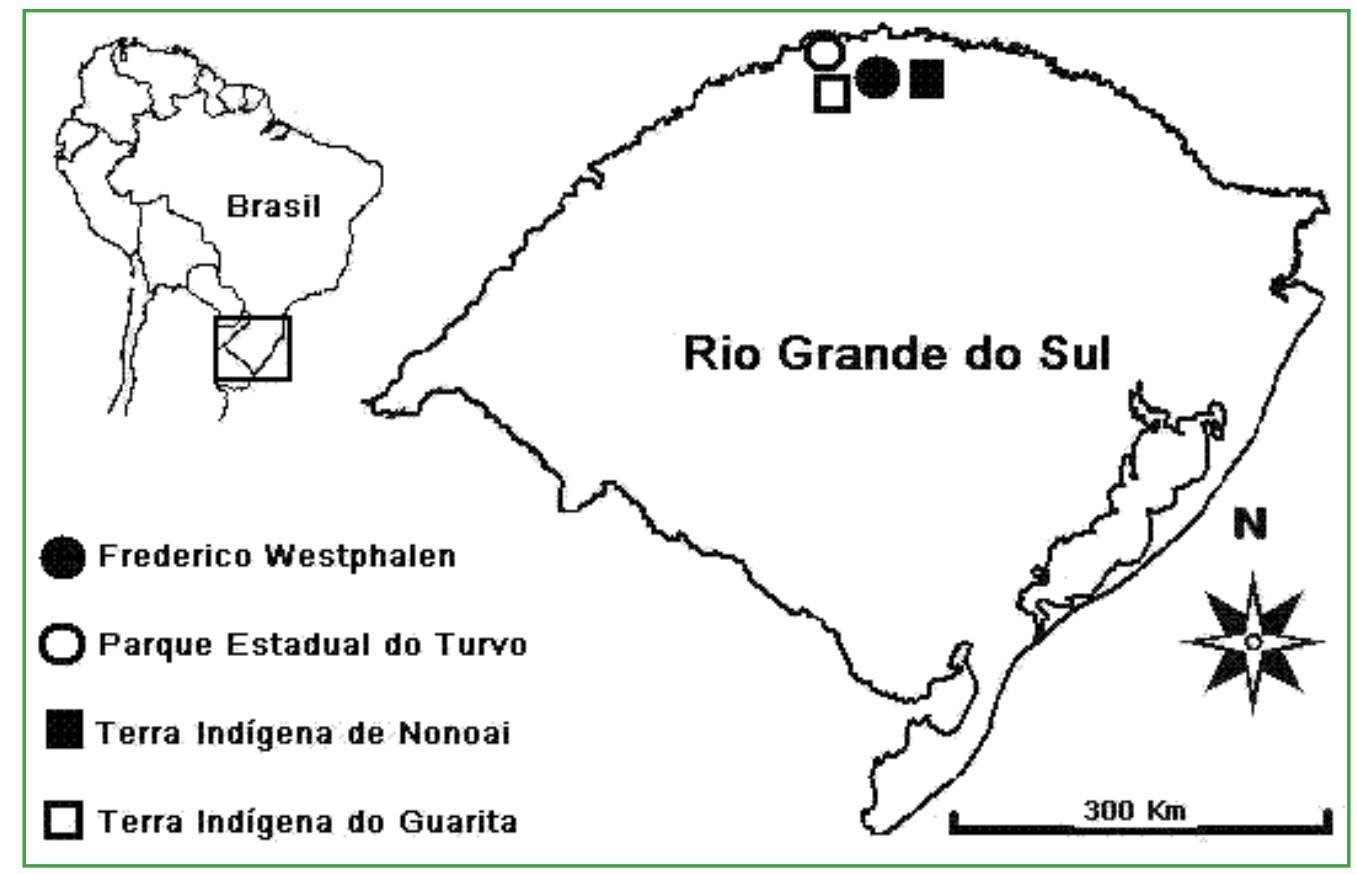

FIGURA 1: Localização do município de Frederico Westphalen, entre os grandes remanescentes florestais do norte do estado. 
$\mathrm{O}$ ordenamento taxonômico e nomenclatura das espécies seguem a Lista das Aves do Brasil do Comitê Brasileiro de Registros Ornitológicos - BRO (2008).

\section{Resultados e Discussão}

Foram registradas 165 espécies de aves pertencentes a 51 famílias (Tabela 1), o que corresponde a $26,4 \%$ das 624 espécies citadas por Bencke (2001) para o Rio Grande do Sul.

TABELA 1: Lista de espécies de aves registradas no município de Frederico Westphalen, RS. Status de ameaça segue Bencke et al. (2003). VU = Vulnerável, $C R=$ Criticamente em perigo, $\mathrm{DD}=$ Dados insuficientes. $?=$ ver resultados e discussão.

\begin{tabular}{|c|c|c|}
\hline Família & Espécie & Status de ameaça \\
\hline \multirow[t]{2}{*}{ Tinamidae } & Crypturellus obsoletus (Temminck, 1815) & - \\
\hline & Nothura maculosa (Temminck, 1815) & - \\
\hline \multirow[t]{2}{*}{ Anatidae } & Dendrocygna viduata (Linnaeus, 1766) & - \\
\hline & Amazonetta brasiliensis (Gmelin, 1789) & - \\
\hline Cracidae & Penelope obscura Temminck, 1815 & - \\
\hline Phalacrocoracidae & Phalacrocorax brasilianus (Gmelin, 1789) & - \\
\hline Anhingidae & Anhinga anhinga (Linnaeus, 1766) & - \\
\hline \multirow{6}{*}{ Ardeidae } & Ardea cocoi Linnaeus, 1766 & - \\
\hline & Bubulcus ibis (Linnaeus, 1758) & - \\
\hline & Ardea alba Linnaeus, 1758 & - \\
\hline & Egretta thula (Molina, 1782) & - \\
\hline & Butorides striata (Linnaeus, 1758) & - \\
\hline & Nycticorax nycticorax (Linnaeus, 1758) & - \\
\hline \multirow[t]{2}{*}{ Threskiornithidae } & Phimosus infuscatus (Lichtenstein, 1823) & - \\
\hline & Plegadis chihi (Vieillot, 1817) & - \\
\hline \multirow[t]{3}{*}{ Cathartidae } & Cathartes aura (Linnaeus, 1758) & - \\
\hline & Coragyps atratus (Bechstein, 1793) & - \\
\hline & Sarcoramphus papa (Linnaeus, 1758) & $\mathrm{CR}$ \\
\hline \multirow[t]{7}{*}{ Accipitridae } & Accipiter striatus Vieillot, 1808 & - \\
\hline & Accipiter bicolor (Vieillot, 1817) & - \\
\hline & Elanoides forficatus (Linnaeus, 1758) & - \\
\hline & Elanus leucurus (Vieillot, 1818) & - \\
\hline & Ictinia plumbea (Gmelin, 1788) & - \\
\hline & Rupornis magnirostris (Gmelin, 1788) & - \\
\hline & Buteo brachyurus Vieillot, 1816 & - \\
\hline \multirow[t]{4}{*}{ Falconidae } & Caracara plancus (Muller, 1777) & - \\
\hline & Milvago chimachima (Vieillot, 1816) & - \\
\hline & Falco sparverius Linnaeus, 1758 & - \\
\hline & Falco peregrinus Tunstall, 1771 & - \\
\hline \multirow[t]{4}{*}{ Rallidae } & Aramides cajanea (Statius Muller, 1776) & - \\
\hline & Aramides saracura (Spix, 1825) & - \\
\hline & Gallinula chloropus (Linnaeus, 1758) & - \\
\hline & Porphyrio martinica (Linnaeus, 1766) & - \\
\hline Charadriidae & Vanellus chilensis (Molina, 1782) & - \\
\hline
\end{tabular}




\begin{tabular}{|c|c|c|}
\hline Recurvirostridae & Himantopus melanurus Vieillot, 1817 & - \\
\hline Scolopacidae & Tringa flavipes (Gmelin, 1789) & - \\
\hline Jacanidae & Jacana jacana (Linnaeus, 1766) & - \\
\hline Columbidae & $\begin{array}{l}\text { Columbina talpacoti } \text { (Temminck, 1811) } \\
\text { Columbina picui (Temminck, 1813) } \\
\text { Columba livia Gmelin, 1789 } \\
\text { Patagioenas picazuro (Temminck, 1813) } \\
\text { Zenaida auriculata (Dês Murs, 1847) } \\
\text { Leptotila verreauxi Bonaparte, } 1855\end{array}$ & $\begin{array}{l}- \\
- \\
- \\
- \\
- \\
-\end{array}$ \\
\hline Psittacidae & $\begin{array}{l}\text { Pyrrhura frontalis (Vieillot, 1817) } \\
\text { Myiopsitta monachus (Boddaert, 1783) } \\
\text { Pionus maximiliani (Khul, 1820) } \\
\text { Melopsittacus undulatus (Shaw, 1805) }\end{array}$ & $\begin{array}{l}- \\
- \\
- \\
-\end{array}$ \\
\hline Cuculidae & $\begin{array}{l}\text { Coccyzus melacoryphus Vieillot, } 1817 \\
\text { Piaya cayana (Linnaeus, 1766) } \\
\text { Crotophaga major Gmelin, 1788 } \\
\text { Crotophaga ani Linnaeus, } 1758 \\
\text { Guira guira (Gmelin, 1788) }\end{array}$ & $\begin{array}{c}- \\
- \\
\text { VU } \\
- \\
-\end{array}$ \\
\hline Tytonidae & Tyto alba (Scopoli, 1769) & - \\
\hline Strigidae & $\begin{array}{l}\text { Rhinoptynx clamator (Vieillot, 1808) } \\
\text { Megascops choliba (Vieillot, 1817) } \\
\text { Pulsatrix koeniswaldiana (Bertoni \& Bertoni, 1901) } \\
\text { Strix hylophyla Temminck, } 1825 \\
\text { Glaucidium brasilianum (Gmelin, 1788) } \\
\text { Athene cunicularia (Molina, 1782) } \\
\text { Asio stygius (Wagler, 1832) }\end{array}$ & $\begin{array}{c}- \\
- \\
\text { DD } \\
- \\
- \\
- \\
\text { DD }\end{array}$ \\
\hline
\end{tabular}

\begin{tabular}{|c|c|c|}
\hline Nyctibiidae & Nyctibius griseus (Gmelin, 1789) & - \\
\hline Caprimulgidae & $\begin{array}{l}\text { Lurocalis semitorquatus (Gmelin, 1789) } \\
\text { Podager nacunda (Vieillot, 1817) } \\
\text { Nyctidromus albicollis (Gmelin, 1789) } \\
\text { Caprimulgus parvulus Gould,1837 } \\
\text { Hydropsalis torquata (Gmelin, 1789) }\end{array}$ & $\begin{array}{l}- \\
- \\
- \\
-\end{array}$ \\
\hline Apodidae & Chaetura meridionalis Hellmayr, 1907 & - \\
\hline Trochilidae & $\begin{array}{l}\text { Phaethornis eurynome (Lesson, 1832) } \\
\text { Florisuga fusca (Vieillot, 1817) } \\
\text { Anthracothorax nigricollis (Vieillot, 1817) } \\
\text { Stephanoxis lalandi (Vieillot, 1818) } \\
\text { Chlorostilbon lucidus (Shaw, 1812) } \\
\text { Hylocharis chrysura (Shaw, 1812) } \\
\text { Leucochloris albicollis (Vieillot, 1818) } \\
\text { Heliomaster furcifer (Shaw, 1812) } \\
\text { Calliphlox amethystina (Boddaert, 1783) }\end{array}$ & $\begin{array}{l}\text { VU } \\
- \\
- \\
- \\
- \\
- \\
- \\
\text { DD } \\
\text { DD }\end{array}$ \\
\hline Trogonidae & Trogon surrucura Vieillot, 1817 & - \\
\hline Alcedinidae & $\begin{array}{l}\text { Ceryle torquatus (Linnaeus, 1766) } \\
\text { Chloroceryle amazona (Latham, 1790) }\end{array}$ & - \\
\hline
\end{tabular}


Chloroceryle americana (Gmelin, 1788)

\begin{tabular}{|c|c|c|}
\hline Bucconidae & Nystalus chacuru (Vieillot, 1816) & - \\
\hline Ramphastidae & $\begin{array}{l}\text { Ramphastos dicolorus Linnaeus, } 1766 \\
\text { Pteroglossus bailloni (Vieillot, 1819) }\end{array}$ & $\begin{array}{c}- \\
\mathrm{CR}\end{array}$ \\
\hline Picidae & $\begin{array}{l}\text { Picumnus temminckii Lafresnaye, } 1845 \\
\text { Melanerpes candidus (Otto, 1796) } \\
\text { Veniliornis spilogaster (Wagler, 1827) } \\
\text { Piculus aurulentus (Temminck, 1821) } \\
\text { Colaptes melanochloros (Gmelin, 1788) } \\
\text { Colaptes campestris (Vieillot, 1818) } \\
\text { Dryocopus sp. }\end{array}$ & $\begin{array}{l}- \\
- \\
- \\
- \\
- \\
- \\
?\end{array}$ \\
\hline Thamnophilidae & $\begin{array}{l}\text { Batara cinerea (Vieillot, 1819) } \\
\text { Thamnophilus caerulescens Vieillot, } 1816\end{array}$ & $\begin{array}{l}- \\
-\end{array}$ \\
\hline Scleruridae & Sclerurus scansor (Ménétriès, 1835) & - \\
\hline Dendrocolaptidae & $\begin{array}{l}\text { Dendrocolaptes platyrostris Spix, } 1825 \\
\text { Campylorhamphus falcularius (Vieillot, 1822) } \\
\text { Lepidocolaptes falcinellus (Cabanis \& Heine, 1859) }\end{array}$ & $\begin{array}{l}- \\
- \\
-\end{array}$ \\
\hline Furnariidae & $\begin{array}{l}\text { Furnarius rufus (Gmelin, 1788) } \\
\text { Lochmias nematura (Lichtenstein, 1823) }\end{array}$ & $\begin{array}{l}- \\
-\end{array}$ \\
\hline Tyrannidae & $\begin{array}{l}\text { Elaenia flavogaster (Thunberg, 1822) } \\
\text { Phylloscartes ventralis (Temminck, 1824) } \\
\text { Myiornis auricularis (Vieillot, 1818) } \\
\text { Myiophobus fasciatus (Statius Muller, 1776) } \\
\text { Pyrocephalus rubinus (Boddaert, 1783) } \\
\text { Machetornis rixosa (Vieillot, 1819) } \\
\text { Pitangus sulphuratus (Linnaeus, 1766) } \\
\text { Myiodynastes maculatus (Statius Muller, 1776) } \\
\text { Megarynchus pitangua (Linnaeus, 1766) } \\
\text { Empidonomus varius (Vieillot, 1818) } \\
\text { Tyrannus melancholicus Vieillot, 1819 } \\
\text { Tyrannus savana Vieillot, } 1808\end{array}$ & $\begin{array}{l}- \\
- \\
- \\
- \\
- \\
- \\
- \\
- \\
- \\
- \\
-\end{array}$ \\
\hline Cotingidae & Pyroderus scutatus (Shaw, 1792) & $\mathrm{CR}$ \\
\hline Pipridae & Chiroxiphia caudata (Shaw \& Nodder, 1793) & - \\
\hline Tityridae & Tityra cayana (Linnaeus, 1766) & - \\
\hline Vireonidae & $\begin{array}{l}\text { Cyclarhis gujanensis (Gmelin, 1789) } \\
\text { Vireo olivaceus (Linnaeus, 1766) }\end{array}$ & - \\
\hline Corvidae & Cyanocorax chrysops (Vieillot, 1818) & - \\
\hline Hirundinidae & $\begin{array}{l}\text { Tachycineta albiventer (Boddaert, 1783) } \\
\text { Tachycineta leucorrhoa (Vieillot, 1817) } \\
\text { Progne tapera (Vieillot, 1817) } \\
\text { Progne chalybea (Gmelin, 1789) } \\
\text { Pygochelidon cyanoleuca (Vieillot, 1817) } \\
\text { Stelgidopteryx ruficollis (Vieillot, 1817) }\end{array}$ & $\begin{array}{l}- \\
- \\
- \\
- \\
-\end{array}$ \\
\hline Troglodytidae & Troglodytes musculus Naumann, 1823 & - \\
\hline
\end{tabular}


Turdidae

Turdus rufiventris Vieillot, 1818

Turdus leucomelas Vieillot, 1818

Turdus amaurochalinus Cabanis, 1850

Turdus albicollis Vieillot, 1818

\begin{tabular}{|c|c|c|}
\hline Mimidae & Mimus saturninus (Lichtenstein, 1823) & - \\
\hline Motacillidae & Anthus lutescens Pucheran, 1855 & - \\
\hline Thraupidae & $\begin{array}{l}\text { Pyrrhocoma ruficeps (Strickland, 1844) } \\
\text { Trichothraupis melanops (Vieillot, 1818) } \\
\text { Tachyphonus coronatus (Vieillot, 1822) } \\
\text { Thraupis sayaca (Linnaeus, 1766) } \\
\text { Thraupis bonariensis (Gmelin, 1789) } \\
\text { Pipraeidea melanonota (Vieillot, 1819) } \\
\text { Tangara preciosa (Cabanis, 1850) } \\
\text { Tersina viridis (Illiger, 1811) } \\
\text { Dacnis cayana (Linnaeus, 1766) } \\
\text { Hemithraupis guira (Linnaeus, 1766) } \\
\text { Conirostrum speciosum (Temminck, 1824) }\end{array}$ & $\begin{array}{l}- \\
- \\
- \\
- \\
- \\
- \\
- \\
- \\
- \\
-\end{array}$ \\
\hline Emberizidae & $\begin{array}{l}\text { Zonotrichia capensis (Statius Muller, 1776) } \\
\text { Poospiza lateralis (Nordmann, 1835) } \\
\text { Sicalis flaveola (Linnaeus, 1766) } \\
\text { Embernagra platensis (Gmelin, 1789) } \\
\text { Volatinia jacarina (Linnaeus, 1766) } \\
\text { Sporophila caerulescens (Vieillot, 1823) } \\
\text { Coryphospingus cucullatus (Statius Muller, 1776) } \\
\text { Paroaria coronata (Miller, 1776) }\end{array}$ & $\begin{array}{l}- \\
- \\
- \\
- \\
- \\
- \\
- \\
-\end{array}$ \\
\hline Cardinalidae & $\begin{array}{l}\text { Saltator similis (d'Orbigny \& Lafresnaye, 1837) } \\
\text { Cyanocompsa brissonii (Lichtenstein, 1823) }\end{array}$ & - \\
\hline Parulidae & $\begin{array}{l}\text { Parula pitiayumi (Vieillot, 1817) } \\
\text { Geothlypis aequinoctialis (Gmelin, 1789) } \\
\text { Basileuterus culicivorus (Deppe, 1830) } \\
\text { Basileuterus leucoblepharus (Vieillot, 1817) }\end{array}$ & $\begin{array}{l}- \\
- \\
- \\
-\end{array}$ \\
\hline Ictiridae & $\begin{array}{l}\text { Cacicus chrysopterus (Vigors, 1825) } \\
\text { Cacicus haemorrhous (Linnaeus, 1766) } \\
\text { Icterus cayanensis (Linnaeus, 1766) } \\
\text { Agelaioides badius (Vieillot, 1819) } \\
\text { Molothrus bonariensis (Vieillot, 1789) } \\
\text { Sturnella superciliaris (Bonaparte, 1850) }\end{array}$ & $\begin{array}{l}- \\
- \\
- \\
-\end{array}$ \\
\hline Fringillidae & $\begin{array}{l}\text { Carduelis magellanica (Vieillot, 1805) } \\
\text { Euphonia clorotica (Linnaeus, 1766) } \\
\text { Euphonia chalybea (Mikan, 1825) } \\
\text { Chlorophonia cyanea (Thunberg, 1822) }\end{array}$ & $\begin{array}{l}- \\
- \\
-\end{array}$ \\
\hline Passeridae & Passer domesticus (Linnaeus, 1758) & - \\
\hline
\end{tabular}


A riqueza de aves observada em Frederico Westphalen é bastante expressiva se considerarmos que os registros foram realizados em um mosaico de pequenos fragmentos florestais, áreas de cultivo, pastagens ou mesmo em ambiente urbano. Provavelmente a localização da área de estudo entre os três grandes remanescentes florestais no norte do estado influenciou positivamente a riqueza observada.

Não foi possível atribuir epíteto específico ao exemplar de Dryocopus (Picidae) observado, entretanto, duas espécies do referido gênero possuem registros para esta porção do estado: D. galeatus (Temminck, 1822) possui registros para o Parque Estadual do Turvo - PET e status de ameaçado sob a categoria "Criticamente em Perigo" no Rio Grande do Sul (Bencke et al., 2003); e $D$. lineatus (Linnaeus, 1766), que possui registros para o PET e para as Terras Indígenas do Guarita - TIG e de Nonoai - TIN além de outras localidades do estado e possui status de ameaçado sob a categoria "Vulnerável" no estado (Bencke et al., 2003).

Para a porção norte do estado merecem destaque na presente lista Asio stygius, registrada no Parque Estadual do Espigão Alto - PEEA, Pulsatrix koeniswaldiana registrada no PET, PEEA e Parque Estadual de Rondinha - PER (Bencke et al., 2003), Calliphlox amethystina e Heliomaster furcifer inseridas na categoria "Dados Insuficientes" (DD) (Bencke et al., 2003), Phaethornis eurynome, registrada no PET (Albuquerque, 1981), TIN e TIG (Bencke et al., 2003), e Crotophaga major, registrada no PET, Derrubadas e Criciumal, na categoria ameaçado - "Vulnerável" (VU) (Bencke et al., 2003), Sarcoramphus papa, registrada no PET (Mähler Jr., 1996), Pyroderus scutatus, registrada no PET (Albuquerque, 1981) e TIN (Bencke et al., 2003), Pteroglossus baillonius (=Baillonius bailloni), registrada no PET (Albuquerque, 1981) e na TIN (Belton, 1994), na categoria "Criticamente em Perigo" (CR) (Bencke et al., 2003) e Glaucidium brasilianum, registrada no PET (Albuquerque, 1981), considerada rara no Rio Grande do Sul (Belton, 1994; Bencke, 2001), como já apresentado com maiores detalhes por Bernardi et al. (no prelo). A floresta estacional do Alto Uruguai, juntamente com a floresta atlântica de planície do litoral norte são os hábitats florestais do estado que concentram o maior número de espécies de aves ameaçadas (Bencke et al., 2003).

Espécies registradas para a porção norte do estado, como: Tinamus solitarius com registros no PET (Albuquerque, 1981) e no PEEA (Bencke et al. 2003), Mesembrinibis cayennensis registrada no PET (Mähler Jr., 1996), Leptodon cayanensis registrada no PET e na TIN (Belton, 1994), Accipiter poliogaster registrada no PET (Mähler Jr., 1996), Spizaetus melanoleucus registrada no PET (Bencke, 2001), Spizaetus tyrannus registrada no PET (Mähler Jr., 1996), Cairina moschata registrada no PET (Albuquerque, 1981) e na TIN (Bencke et al. 2003), Sarkidiornis melanotos registrada no PET (Albuquerque, 1981), Aburria jacutinga registrada no PET (Albuquerque, 1981) e na TIN (Belton, 1994), Odontophorus capueira e Patagioenas cayennensis registradas no PET (Albuquerque, 1981) e no PEEA (Bencke et al. 2003), Patagioenas plumbea e Claravis pretiosa registradas no PET (Albuquerque, 1981), Dromococcyx phasianellus registrada no PET (Albuquerque, 1981) e em Iraí (Belton, 1994), Strix virgata registrada no PET (IPB com. pess.), Caprimulgus sericocaudatus registrada no PET (Bencke, 2001), Baryphthengus ruficapillus registrada no PET, na TIN e no PER (Belton, 1994), Pteroglossus castanotis registrada no PET e na TIN (Bencke et al. 2003), Selenidera maculirostris registrada no PET (Mähler Jr., 1996) e na TIN (Belton, 1994), Ramphastos toco registrada no PET (Albuquerque, 1981), Campephilus robustus registrada no PET (Albuquerque, 1981), na TIN e no PER (Bencke et al., 2003), Dendrocincla turdina registrada no PET (Bencke et al., 2003), Clibanornis dendrocolaptoides registrada no PET (Mähler Jr., 1996), Philydor lichtensteini registrada no PET (Albuquerque, 1981), na TIN e em Iraí (Bencke et al., 2003), Mackenziaena severa registrada no PET (Albuquerque, 1981), na TIN (Belton, 1994) e em Iraí (Bencke et al., 2003), Drymophila rubricollis registrada no PET (Albuquerque, 1981), na TIN (Belton, 1994) e no PEEA (Bencke et al., 2003), Pyriglena leucoptera registrada no PET (Albuquerque, 1981) e no PEEA (Bencke et al., 2003), Grallaria varia registrada no PET (Albuquerque, 1981) e na TIN (Bencke et al., 2003), Phylloscartes eximius registrada no PET, PER, PEEA e na TIN (Bencke et al., 2003), Capsiempis flaveola registrada no PET 
(Albuquerque, 1981), na TIN, na TIG e em Três Passos (Bencke et al., 2003), Corythopis delalandi registrada no PET (Albuquerque, 1981), na TIN (Belton, 1994), na TIG, em Erval Seco e em Tiradentes do Sul (Bencke et al., 2003), Hemitriccus diops registrada em Iraí (Belton, 1994) e na TIN (Bencke et al., 2003), Contopus cinereus registrado no PET, na TIN e no PEEA (Bencke et al., 2003), Colonia colonus registrada no PET (Albuquerque, 1981) e na TIN (Belton, 1994), Piprites chloris registrada no PET e na TIN (Belton, 1994), Phibalura flavirostris registrada no PET (Albuquerque, 1981), PEEA e na TIN (Belton, 1994), Polioptila lactea registrada no PET (Albuquerque, 1981) e na TIN (Belton, 1994) na TIN e em Iraí (Bencke et al., 2003), Sporophila angolensis registrada no PET (Mähler Jr., 1996) e na TIN (Belton, 1994), Saltator fuliginosus registrada no PET (Albuquerque, 1981), Cissopis leverianus registrada no PET e na TIN (Bencke et al., 2003), Nemosia pileata registrada no PET (Belton, 1994), Euphonia violacea registrada no PET (Albuquerque, 1981) e no PEEA (Bencke et al., 2003), Tangara seledon registrada no PET (Albuquerque, 1981), Cacicus solitarius registrada no PET (Mähler Jr., 1996) e na TIN ((Bencke, 2001), e Molothrus oryzivorus registrada no PET (Bencke, 2001), arredores da TIG (Bencke et al., 2003) e nas proximidades de Tenente Portela (Belton, 1994), também sofrem algum grau de ameaça e não foram registradas no município.

Das 247 espécies registradas por Albuquerque (1981) e Mähler Jr. (1996), nos únicos trabalhos publicados realizados no extremo norte do estado, 117 espécies não foram registradas em Frederico Westphalen, e 35 espécies registradas nesse estudo não foram registradas por esses autores. Merecem destaque: Falco peregrinus, Aramides cajanea, Myiopsitta monachus, Rhinoptynx clamator, Podager nacunda, Florisuga fusca e Hylocharis chrysura, não citadas por Belton (1994) para o extremo norte do estado nem registradas por Albuquerque (1981) e Mähler Jr. (1996), e que também não possuem registros publicados para essa porção do estado.

A continuidade e melhor distribuição dos esforços (a porção norte do município foi pouco amostrada) e o emprego de métodos mais rigorosos (e.g. capturas com redes de neblina) que permitem amostrar espécies de difícil identificação e visualização deverão aumentar significativamente a lista ora apresentada para Frederico Westphalen.

\section{Agradecimentos}

Os autores agradecem aos proprietários das áreas particulares por permitirem o acesso, a Glayson A. Bencke pela confirmação da identificação de algumas espécies e leitura crítica dos originais e aos dois consultores anônimos pelas correções e sugestões.

\section{Referências}

Albuquerque, E. P. 1981. Lista preliminar das aves observadas no Parque Florestal Estadual do Turvo, Tenente Portela, Rio Grande do Sul, Brasil. Roessleria, 4: 107-122.

Belton, W. 1984. Birds of Rio Grande do Sul, Brazil, Part 1. Rheidae through Furnariidae. Bulletin of American Museum of Natural History, 178: 369-636.

Belton, W. 1994. Aves do Rio Grande do Sul, distribuição e biologia. Ed. Unisinos, São Leopoldo, Brasil, 584pp.

Bencke, G. A. 2001. Lista de referência das aves do Rio Grande do Sul. Fundação Zoobotânica do Rio Grande do Sul, Porto Alegre, Brasil, 104pp.

Bencke, G. A.; Fontana, C. S.; Dias, R. A.; Maurício, G. N.; Mähler Jr., J. K. F. 2003. Aves. In: Fontana, C. S.; Bencke, G. A. \& Reis, R. E. (Eds). Livro vermelho da fauna ameaçada de extinção no Rio Grande do Sul. EDIPUCRS, Porto Alegre, Brasil, p.189-489.

Bernardi, I. P.; Pulchério-Leite, A.; Miranda, J. M. D.; Passos, F. C. 2007. Ampliação da distribuição de Molossops neglectus Williams e Genoways (Chiroptera, Molossidae) para o Sul da América do Sul. Revista Brasileira de Zoologia, 24: 505-507.

Bernardi, I. P.; Teixeira, E. M.; Jacomassa, F. A. F. (no prelo). Registros relevantes da avifauna do Alto Uruguai, Rio Grande do Sul, Brasil. Biociências.

CBRO, 2008. Lista das Aves do Brasil. Disponível em <http:// www.cbro.org.br>. Acesso em 03 de novembro de 2008.

Irgang, B. E. 1980. A mata do Alto Uruguai no RS. Ciência e Cultura, 32: 323-324.

Mähler Jr., J. K. F. 1996. Contribuição ao conhecimento da avifauna do Parque Estadual do Turvo, Rio Grande do Sul, Brasil. Acta Biologica Leopoldensia, 18: 123-128. 